These observations show many parallels with those for Trifolium repens (Daday 1954a, b) and Lotus corniculatus (Jones 1977), where the existence of a genetically determined polymorphism, sometimes modified by developmental and environmental factors, has been proposed. The stable polymorphic mosaics in bracken are most easily explained in the same way. However, a genetic basis for the polymorphism in bracken has not yet been confirmed, and additional, preliminary, results include the observations that (i) rhizomes producing acyanogenic fronds in the field produced cyanogenic fronds the following season when transplanted into garden plots, and (ii) spores collected from acyanogenic fronds in the field produced cyanogenic sporelings in laboratory cultures. These turned acyanogenic when they became pot bound, as did sporelings raised from spores produced by cyanogenic fronds.

These suggest that the physiological state of the rhizomes or individual frond buds may be important in influencing the expression of the cyanogenic genotype in the fronds which develop from them. Thus, local variations in light, nutrient or water availability may influence the cyanogenic phenotype. It may even be that acyanogenic genotypes do not exist in bracken. The causes of the polymorphism are currently being investigated. An understanding of the underlying mechanism is needed in order to assess the ecological role, if any, of cyanogenesis, and the possibilities of using cyanogenesis as a genetic marker for the study of bracken population structure. This in turn has important implications for strategies of bracken control.

This work is supported by a NERC CASE studentship to P.R.H.H.

Conn, E. E. 1980. Cyanogenic glycosides. In Encyclopaedia of Plant Physiology, ed. Bell, E. A. and Charlwood, B. V., Vol. 8, pp. 461-492. Berlin: Springer.

Cooper-Driver, G. and Swain, T. 1976. Cyanogenic polymorphism in bracken in relation to herbivore predation. Nature 260, 604.

Daday, H. 1954a. Gene frequencies in wild populations of Trifolium repens. I. Distribution by latitude. Heredity 8, 61-78.

- 1954b. Gene frequencies in wild populations of Trifolium repens. II. Distribution by altitude. Heredity 8, 377-384.

Fiegl, F. and Anger, V. 1966. Replacement of benzidine by copper ethyl acetoacetate and tetrabase as spot test reagent for Hydrogen cyanide and cyanogen. Analyst 91, 282-284.

Greshoff, M. 1908. Transitorische Blausäure in Farnen. Pharm. Weekbl. Ned. 45, 770-773.

Jones, D. A. 1966. On the polymorphism of cyanogenesis in Lotus corniculatus I. Selection by animals. Can. J. Genet. 8, 556-567.

— 1977. On the polymorphism of cyanogenesis in Lotus corniculatus VII. The distribution of the cyanogenic form in Western Europe. Heredity 39, 27-44.

Lawton, J. H. 1976. The structure of the arthropod community on bracken. Bot. J. Linn. Soc. 73, 187-216.

\title{
Effect of G418 on spore germination and growth of Pteridium aquilinum
}

\author{
J. M. S. Jubrael and E. Sheffield \\ Department of Botany, University of Manchester, Oxford Road, \\ Manchester M13 9PL, U.K.
}

G418 is an antibiotic produced by Micromonospora rhodorangea. It has been found to have an inhibitory effect on a wide variety of prokaryotic and eukaryotic organisms but has not previously been tested on pteridophytes. The structure and chemical 
stability have been described by Daniels et al. (1973). It is an aminoglycoside akin to gentamycin and kanamycin. Unlike other aminoglycosides, G418 has an inhibitory effect on eukaryotes (Jimenez and Davies 1980). We hope to develop a fern transformation system and intend to employ G418 as an aid to selection of transformed individuals of $P$. aquilinum.

G418 effects on germination were assessed by sowing spores on plates of Moore's medium containing 0 to $2,000 \mu \mathrm{g} / \mathrm{ml} \mathrm{G418}$. The effects on growth were monitored using germinated spores. Three-day-old gametophytes were plated on medium containing 0 to $100 \mu \mathrm{g} / \mathrm{ml} \mathrm{G} 418$.

The results showed that G418 has a strong inhibitory effect on both germination and growth in Pteridium. It is effective at very low concentrations: $50 \mu \mathrm{g} / \mathrm{ml}$ is sufficient to prevent both germination and growth.

The mode of action has not yet been elucidated, but G418 is clearly an excellent candidate for use in eukaryotic selection systems, as transformed individuals will be easily identified by their ability to grow on media containing $50 \mu \mathrm{g} / \mathrm{ml} \mathrm{G} 418$ or above.

Daniels, P. J. L., Yehaskel, A. S. and Morton, J. 1973. The structure of antibiotic G418. 13th Interscience Conf. Antimicrobial Agents and Chemotherapy, Washington. Abstract 137.

Jimenez, A. and Davies, J. 1980. Expression of a transposable antibiotic resistance element in Saccharomyces. Nature $287,869-871$.

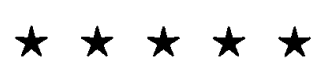

\title{
E. EXPERIMENTAL STUDIES ON FERN-ALLIES
}

\author{
Spore germination in Psilotum \\ Dean P. Whittier \\ Department of General Biology, Vanderbilt University, Nashville, \\ TN 37235, U.S.A.
}

Previous attempts at germinating the spores of Psilotum resulted in less than $0.1 \%$ germination after 6 months in the dark (light prevents germination). These experiments were carried out on a nutrient medium containing Knudson's solution of mineral salts which contains both nitrate and ammonium ions, minor elements, FeEDTA, $0.5 \%$ sucrose, and $0.6 \%$ agar. The form of the nitrogen in the medium greatly affects germination. Media which contain nitrate $\left(50 \mathrm{mgl}^{-1}\right)$ with or without ammonium ions inhibit germination. Spore germination on media with 50 $0.05 \mathrm{mgl}^{-1}$ nitrate increases as the nitrate concentration decreases. Germination is excellent for spores cultured on media containing ammonium ions as the nitrogen source or a medium without nitrogen. Tests with several ammonium salts, supplying $50 \mathrm{mgl}^{-1}$ ammonium ions, give $85-90 \%$ germination. In addition to 